\title{
Beyond Janus Geometry: Characterization of Flow Fields Around Nonspherical Photocatalytic Microswimmers
}

\author{
Sandra Heckel Clemens Bilsing Martin Wittmann Thomas Gemming Lars Büttner Jürgen Czarske \\ Juliane Simmchen*
}

S. Heckel, M. Wittmann, Dr. J. Simmchen

TU Dresden, Chair of Physical Chemistry, Zellescher Weg 19, 01069 Dresden, Germany

Email Address: juliane.simmchen@tu-dresden.de

C.-M. Bilsing, Dr. L. Büttner, Prof. Dr. J. Czarske

TU Dresden, Laboratory for Measurement and Sensor System Technique, Helmholtzstraße 18, 01069

Dresden, Germany

Dr. L. Büttner, Prof. Dr. J. Czarske

Competence Center Biomedical Computational Laser Systms (BIOLAS), Helmholtzstraße 18, 01069 Dres-

den, Germany

Dr. T. Gemming

Leibniz Institute for Solid State and Materials Research Dresden, Helmholtzstraße 20, 01069 Dresden, Germany

Keywords: Microswimmers, Photocatalysis, Flow Fields, Particle Tracking Velocimetry

\begin{abstract}
Catalytic microswimmers that move by a phoretic mechanism in response to a self-induced chemical gradient are often obtained by the design of spherical janus microparticles, which suffer from multi-step fabrication and low yields. Approaches such as irregular particle shapes, local excitation or intrinsic asymmetry are on the rise to facilitate manufacturing, but the effects on the generation of motion remain poorly understood. In this work, single crystalline $\mathrm{BiVO}_{4}$ microswimmers are presented that rely on a strict inherent asymmetry of charge-carrier distribution under illumination. The origin of the asymmetrical flow pattern is elucidated because of the high spatial resolution of measured flow fields around pinned BiVO4 colloids. As a result the flow from oxidative to reductive particle sides was confirmed. Distribution of oxidation and reduction reactions suggests a dominant self-electrophoretic motion mechanism with a source quadrupole as the origin of the induced flows. It is shown that the symmetry of the flow fields is broken by selfshadowing of the particles and synthetic surface defects that impact the photocatalytic activity of the microswimmers. The results demonstrate the complexity of symmetry breaking in nonspherical microswimmers and are leading the way towards understanding of propulsion mechanisms of phoretic colloids of various shapes.
\end{abstract}

\section{Introduction}

The design of artificial active matter, i.e. particles that self-propel in a certain direction, is a challenging yet promising subject with envisioned applications in a range of fields like environmental remediation[1, $2,3]$, sensing[4] and biomedical engineering. [5, 6, 7] Due to the physical laws for fluid flow on the microscale, constant reaction throughput that dissipates energy and reaches out-of-equilibrium conditions is required to induce active motion.[8] A synthetic realization are catalytic microswimmers, which typically drive catalytic peroxide degradation on their surface and consequently are understood to move by an interplay of self-diffusiophoretic and self-electrophoretic forces, depending on the specific morphology and reactions taking place.[9, 10] In a nutshell, interactions between the solute, the swimmer surface and the substrate on which they are moving cause fluid flows around the colloids and subsequently active motion. In order for a preferred motion direction to arise, the catalytic reaction needs to take place asymmetrically over the surface of the microswimmer, which is often achieved by the fabrication of janus particles.[11, 12] The understanding of the emerging flows around microswimmers is essential to elucidate their motion behavior, mechanism and interactions between active and passive colloids. Theoretically, potential flow fields can be calculated as solutions of the Navier-Stokes equations.[13] If incompressible flow is assumed, i.e. fluid motion is induced by solute-surface interactions, force multipoles that result in pusher or puller-type swimmers are solutions of the equations. However, on the surface of catalytic particles, compressible fluid flow may have to be considered, which gives rise to source multipoles or neutral microswimmers, where the fluid motion is determined by chemical flows.[13, 14] Experimental realization of self-induced flow fields around microobjects is challenging, and only few examples exist.[15, 
16, 17] For catalytic polystyrene-platinum (PS-Pt) spheres, Campbell et al. determined pusher-type flow fields with current loops from the equator to the platinum pole of the particles.[15] However, catalytic janus microparticles suffer from multi-step fabrication processes with low yield and reproducibility, which is why alternative, scalable approaches with concepts for inherent asymmetry are being investigated.[18, 19] This arises the question about the corresponding flow conditions around these microswimmers. Despite a variety of experimental shapes for active matter, modelling beyond spherical or spheroidal geometries has barely been approached. Recently, we and others proposed single-component $\mathrm{BiVO}_{4} \mathrm{mi}^{-}$ croswimmers that rely on charge-carrier separation due to a surface heterojunction between catalytically active crystal facets. In different studies, $\mathrm{BiVO}_{4}$ colloids have shown great potential for the investigation of the behavior of single microswimmers, [20] but also active assemblies and interaction with passive particles have been demonstrated.[21, 22] Considering that for such microswimmers neither the prediction of expected flows by established theoretical models, nor their experimental resolution has been approached, the understanding of the rise of active motion through facet selective oxidation and reduction reactions lags behind their well-known janus-shaped counterparts.

Here, we develop an experimentally viable approach to determine flow fields around pinned single-crystalline photocatalytic $\mathrm{BiVO}_{4}$ microswimmers with non-spherical geometry. In order to obtain comparable flow patterns, we first optimize the synthesis of these particles with shape-directing surfactants. By particle tracking velocimetry of passive gold tracer particles around pinned microswimmers, we are able to resolve flow patterns around the swimmers and relate these to the observed behavior under illumination. By averaging flow fields of individual particles, we are able to distinguish common trends from effects related to individual particle morphology.

\section{Characterization of $\mathrm{BiVO}_{4}$ Microparticles}

$\mathrm{BiVO}_{4}$ is available in different morphologies from undefined crystals[23] to shuriken,[22, 20] and spheroids.[21] While for bulk chemical reactions the morphologies of individual colloids is of less importance, to ensure comparable hydrodynamic conditions, a low polydispersity is crucial. To achieve a homogeneous particle distribution, as a first step the synthesis of $\mathrm{BiVO}_{4}$ was optimized. Synthesis was carried out using a hydrothermal reaction, where morphology of the resulting crystallites can be controlled by addition of a capping agent.[24] A well known capping agent for $\mathrm{BiVO}_{4}$ are $\mathrm{Cl}^{-}$ions, which selectively adsorb onto the $\{010\}$ facets and reduce the surface energy. By that, growth of these facets is slowed down and an increase in $\mathrm{Cl}^{-}$concentration by addition of $\mathrm{NaCl}$ results in larger exposed $\{010\}$ facets. [25] It was also reported, that sodium dodecyl sulfate (SDS) is selectively adsorbed on $\{110\}$ facets of $\mathrm{BiVO}_{4}$. [26] Therefore, SDS was applied as a possible capping agent for $\{110\}$ facets of $\mathrm{BiVO}_{4}$. For optimisation, different concentrations of $\mathrm{NaCl}$ and SDS were tested and the effect is displayed in Figure 1a. It can be seen, that an increase in SDS concentration causes the formation of thicker particles with larger exposed $\{110\}$ facets, while an increase in $\mathrm{NaCl}$ concentration leads to thinner platelets with preferential exposure of $\{010\}$ facets. Absorption spectra and X-ray diffractograms (XRD) confirm the monoclinic crystal structure and yield a band gap of $2.49 \mathrm{eV}$ (see Figure S1 and S2).

The active motion of the microparticles was investigated by speed and mean-squared displacement (MSD) analysis under ultraviolet (UV) illumination ( $385 \mathrm{~nm}$ wavelength) in $0.1 \mathrm{wt} \% \mathrm{H}_{2} \mathrm{O}_{2}$ solution. Under these conditions, $\mathrm{H}_{2} \mathrm{O}_{2}$ is decomposed into water and oxygen in two separate half-reactions: Due to chargecarrier separation on the $\mathrm{BiVO}_{4}$ surface, this reaction is assumed to be split into an oxidation on $\{110\}$ facets and a reduction on $\{010\}$ facets, which can be shown by photodeposition experiments (see Figure S3).[23] In Figure 1b, the speed of individual single crystalline particles is shown as a combination of data scatter and a box plot. For comparison, it should be noted that the particles neither show any active motion in dark, illumination-free conditions, nor under illumination in absence of $\mathrm{H}_{2} \mathrm{O}_{2}$. The mean speed of their Brownian motion under these conditions was determined as $(2.1 \pm 0.5) \mu_{\mathrm{m} \mathrm{s}^{-1}}$ and $(2.8 \pm 0.3) \mathrm{\mu m} \mathrm{s}^{-1}$, respectively. Under illumination, we observe a tendency for the particles to reorient themselves: While laying on $\{010\}$ facets under dark conditions, the colloids rotate in respect to the substrate under UV excitation by an angle $\alpha$ of up to $90^{\circ}$, which results in an upright position (see Figure 
1b and Video S1). Once reoriented, the microswimmers move with a tendency for curved trajectories, which is in agreement with observations of previously studied $\mathrm{BiVO}_{4}$ microswimmers with comparable shapes.[20] A mean speed of $(6.59 \pm 2.89) \mathrm{\mu m} \mathrm{s}^{-1}$ can be observed. Analysis of the MSD of an exemplary particle further confirms the active motion of the microswimmers (see Figure $\mathbf{S 4}$ ). Compared to earlier presented morphologies, the activity and speed of these microswimmers is strongly increased, which can be justified by the higher catalytic activity of the single crystaline structures.[20, 21, 22]

\section{Flow Field Analysis}

Prior to flow field analysis, the motion mechanism and resulting expectations for the fluid flow shall be considered. The strong separation of reduction and oxidation reaction on the surface of these colloids suggests a dominant self-electrophoretic motion mechanism. For bimetallic self-electrophoretic rods moving by $\mathrm{H}_{2} \mathrm{O}_{2}$ decomposition, fluid flows from oxidation to reduction site were predicted, which is in agreement with a source dipole field.[27] If these considerations are transferred to the single crystalline $\mathrm{BiVO}_{4}$ particles under illumination, proton flows from $\{110\}$ to $\{010\}$ facets can be expected. Due to the upright orientation of the microswimmers under illumination, two proton sources and two proton sinks are anticipated in the substrate plane where motion is observed, causing a source quadrupole (see Figure 2). However, to result in active motion, the flow pattern around the microswimmers is required to be asymmetric. In janus particles or bimetallic rods, this is caused by different interaction potentials of the individual, chemically different, hemispheres with the solutes.[9] The here discussed particles only consist of a single component, namely $\mathrm{BiVO}_{4}$. Yet, different atoms are exposed on the different facets, which could cause a dependence of the surface-solute interaction.[28] Additionally, synthetic surface defects were identified in SEM images, which render the photocatalytic activity of each particle side unique (see Figure S5). Furthermore, the illumination direction of the samples needs to be considered. In the conducted microscopy experiments, samples were illuminated from the bottom through the objective with a UV LED. Due to the high absorption coefficient of $\mathrm{BiVO}_{4}$ in the UV range, it can be assumed that particle sides which are oriented towards the substrate receive more light excitation and therefore photocatalytic activity than particles sides which are oriented towards the top of the sample.[29] In consequence,

(a)

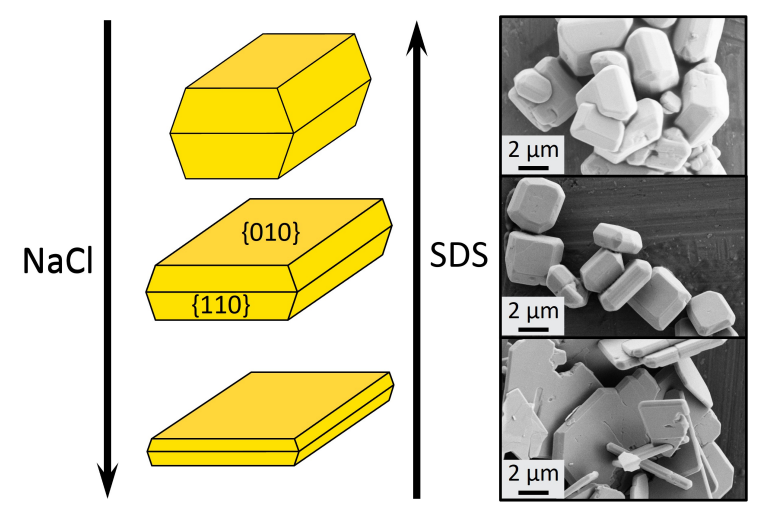

(b)

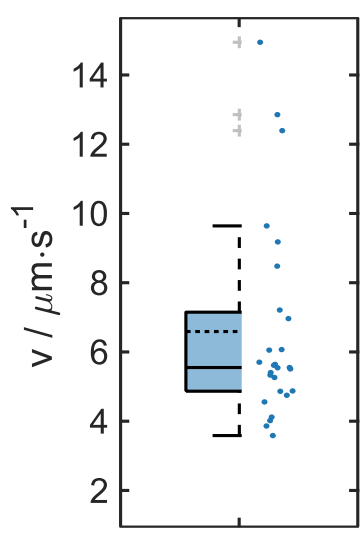

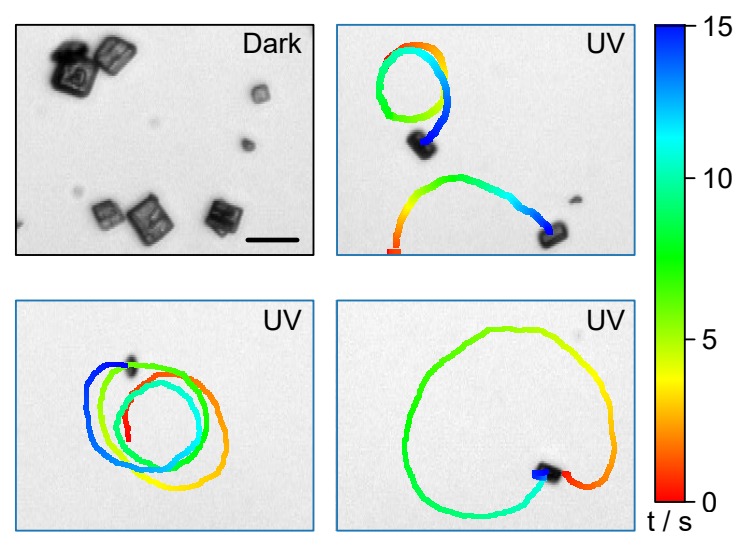

Figure 1: (a) (left) Scheme showing impact of increasing concentration of NaCl and SDS marked by arrows on crystal morphology and (right) SEM images of $\mathrm{BiVO}_{4}$ particles synthesised at different concentrations of $\mathrm{NaCl}$ and SDS: top: $0.05 \mathrm{M}$ $\mathrm{NaCl}$ and $0.05 \mathrm{SDS}$, middle: $0.05 \mathrm{M} \mathrm{NaCl}$ and $0.005 \mathrm{M}$ SDS, bottom: $0.2 \mathrm{M} \mathrm{NaCl}$ and $0.005 \mathrm{M}$ SDS. (b) Speed box plot of single crystalline $\mathrm{BiVO}_{4}$ microswimmers under UV illumination in dilute $\mathrm{H}_{2} \mathrm{O}_{2}$. Solid horizontal line shows median, dashed horizontal line refers to mean. Grey markers refer to outliers, whiskers indicate interquartile range. The blue scatter shows the individual speed values from which the box plot is created. On the right, microscope snapshots show particles in dark conditions, where it can be seen that they lay flat on their $\{010\}$ facet. Under illumination, upright motion with a tendency for circular trajectories is observed. Scale bar is $10 \mu \mathrm{m}$. 
(a)

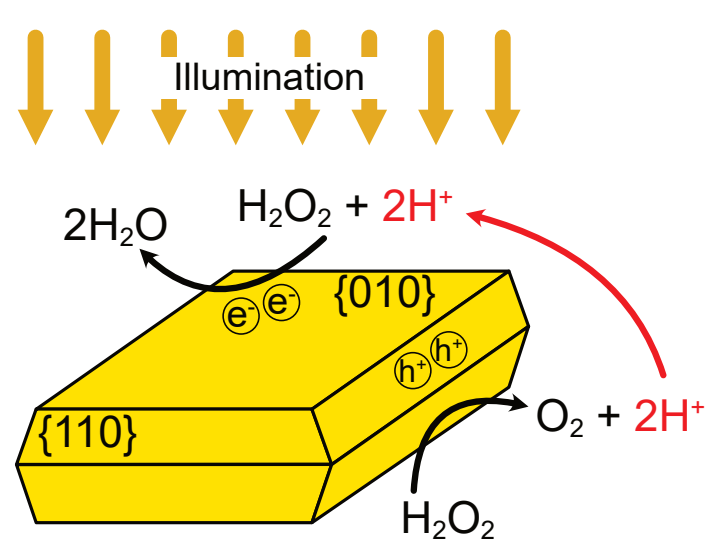

(b)

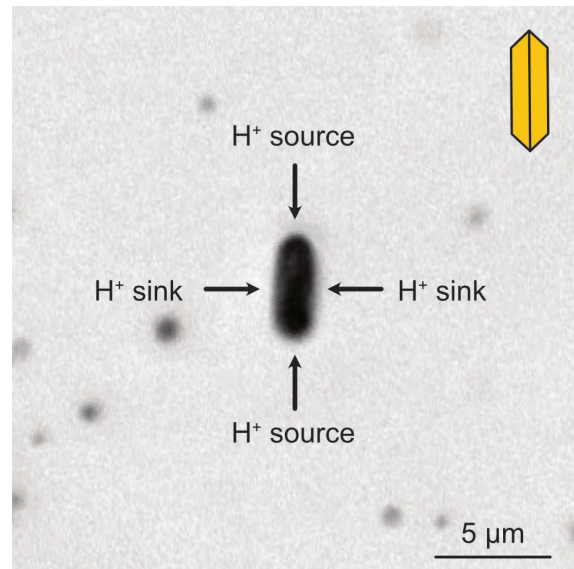

Figure 2: (a) Separation of oxidation and reduction reaction leads to a proton flow from $\{110\}$ to $\{010\}$ facets on the colloid surface under excitation $(\lambda=385 \mathrm{~nm})$. (b) Due to the single crystal morphology, two proton sources and two proton sinks are present in the substrate plane where upright motion is observed.

we assume the self-electrophoretic motion mechanism to be induced by charge-carrier separation onto different facets which leads to proton flows and oxygen gradients under illumination. Additionally, an enhancement of the asymmetry by self-shadowing is expected.

Flow fields were obtained by particle tracking velocimetry (PTV). To ensure good visibility in optical microscopy despite small particle sizes, gold nanoparticles were synthesized as passive tracers, which can be tracked to reconstruct the fluid flow without modifying it significantly by themselves. The quasispherical nanoparticles have a mean diameter of $(250 \pm 22) \mathrm{nm}$ and did not show any catalytic activity towards $\mathrm{H}_{2} \mathrm{O}_{2}$ decomposition (see Figure S6). For video recording, solutions of $0.2 \mathrm{wt} \% \mathrm{H}_{2} \mathrm{O}_{2}$ were prepared and $\mathrm{BiVO}_{4}$ microswimmers as well as Au tracer particles were added. The samples were applied to a glass substrate and subsequently covered with a thin cover glass and sealed to prevent evaporation. Under these conditions, the colloids were confined in samples with a height of $h \approx 30 \mu \mathrm{m}$. To intensify fluid flows, microswimmers were pinned to the glass substrates using high light intensities in upright $\left(\alpha=90^{\circ}\right)$ or inclined $\left(0^{\circ}<\alpha<90^{\circ}\right)$ position which causes them to act as fluid pumps. For video recording, the substrates were inserted in an inverted microscope and illuminated with UV light from the bottom. In PTV analysis, the median speed and motion direction of the tracers was determined for a rasterized display of the surrounding area of the pinned microswimmers (see Figure 6). Details on the implementation can be found in Section 5. The tracer motion can also be seen in Video S2.

Flow fields of two exemplary $\mathrm{BiVO}_{4}$ microparticles can be found in Figure $\mathbf{3 a}$ and $\mathbf{3 b}$. For illustration, the original orientation of an upright and an inclined microswimmer have been added to the flow fields as yellow particle sketches. Additional flow fields can be found in Figure S7. Comparison shows that tracer velocities and motion directions vary between the individual flow fields, especially in the far field. This observation is not surprising, as these microparticles are characterized by synthetic defects that render a unique profile of the spatial resolution of photocatalytic activity for each microswimmer. Additionally, the individual inclination angles towards the substrate influence the illumination of the particle facets. Yet, common trends can be determined. All of the analyzed microparticles exhibit inward flows from their side $(\{110\})$ towards the top and bottom $(\{010\})$ facets, from where the fluid appears to be pushed outward. These results are in agreement with expectations based on the production and consumption sites of $\mathrm{H}^{+}$ions (see Figure 2a). It becomes apparent that the source quadrupole, which is caused by opposite $\{010\}$ facets (along the $x$-axis) and opposite side faces with $\{110\}$ facets (along the $y$-axis) causes a flow field with similarities to a pusher-type force dipole, which indicates that the flow field is overlaid by dominant hydrodynamic interactions. Similar observations have been obtained for fluid flows around immobilized induced-charge electro-osmotic (ICEO) $\mathrm{Au} / \mathrm{SiO}_{2}$ janus particles.[17] Due 
to symmetry axes of the particles and the applied electric field, a quadrupolar fluid flow field is indicated by theoretical considerations. Experimentally, this results in a flow pattern with force dipole similarities whose symmetry is broken by the different polarizability of the two materials.[17]

More detailed information about the flow profiles around the differently oriented microparticles can be obtained by analysis of velocities $u_{x}$ and $u_{y}$ along the $x$ - and $y$-axes of the rastered coordinate system, whose point of origin was placed in the center of the microparticles in each flow field. Therefore, analysis of $u_{x}$ along the $x$-axis enables comparison of flow velocities between the two reductive $\{010\}$ facets. Equivalently, the difference between the oxidative $\{110\}$ facets is characterized by $u_{y}$ along the $y$-axis. Results for the upright oriented particle are shown in Figure $\mathbf{4 a}$ and $\mathbf{4 b}$, and for the inclined particle in Figure $\mathbf{4} \mathbf{c}$ and $\mathbf{4 d}$. The velocities $u_{x}$ and $u_{y}$ were calculated from the respective flow fields by averaging over two raster rows for $u_{x}$ and columns for $u_{y}$. The area covered by the microparticle is indicated by dashed lines in all plots. Interestingly, the tracer flow appears to be maximized at a distance of about $5 \mathrm{~m}$ from the particle surface in all directions, independent of whether the microswimmers are attached in inclined or upright position and along which axis the velocity is analyzed. At higher distances than $5 \mu \mathrm{m}$, the impact of self-induced flows by $\mathrm{BiVO}_{4}$ on the tracers decreases and their motion direction is randomized by Brownian motion, which leads to a decrease of $u_{x}$ and $u_{y}$ towards $0 \mu \mathrm{m} \mathrm{s}^{-1}$. However, differences in $u_{x}$ and $u_{y}$ between the upright and inclined particle are also observed. If $u_{x}$ is compared between the two colloids, it can be seen that mirrored profiles are obtained for the two reductive sides of the upright particle (Figure 4a), with similar maximum velocities of $u_{x} \approx 4 \mu \mathrm{m} \mathrm{s}^{-1}$. For the inclined particle however, this is not the case. While tracers are accelerated to similarly high velocities around $4 \mu \mathrm{m} \mathrm{s}^{-1}$ on its left side, which is oriented towards the glass substrate and therefore also the illumination source, lower values between $1 \mu \mathrm{m} \mathrm{s}^{-1}$ and $-1 \mu \mathrm{m} \mathrm{s}^{-1}$ are obtained on the right side, which points away from the substrate (Figure 4c). Likely, self-shadowing of the right particle side is responsible for the difference observed here. Velocity profiles of $u_{y}$ along the $y$-axis look similar for both particles, with differences of around $1 \mu \mathrm{m} \mathrm{s}^{-1}$ between the two oxidative sides of the colloids (Figure $4 \mathrm{~b}$ and $4 \mathrm{~d}$ ). As excitation from the bottom illuminates these side faces to the same extent independent of the inclination angle, differences in velocity are likely caused by impacts of the unique shape of each particle on its catalytic activity.

In summary, we find that analysis of the tracer velocities supports the prediction, that the asymmet-

(a)

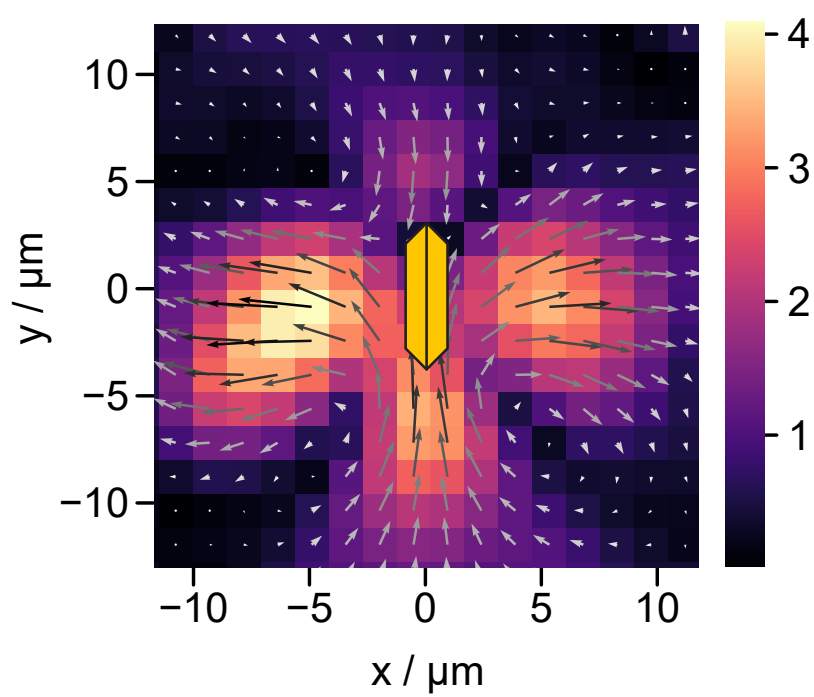

(b)

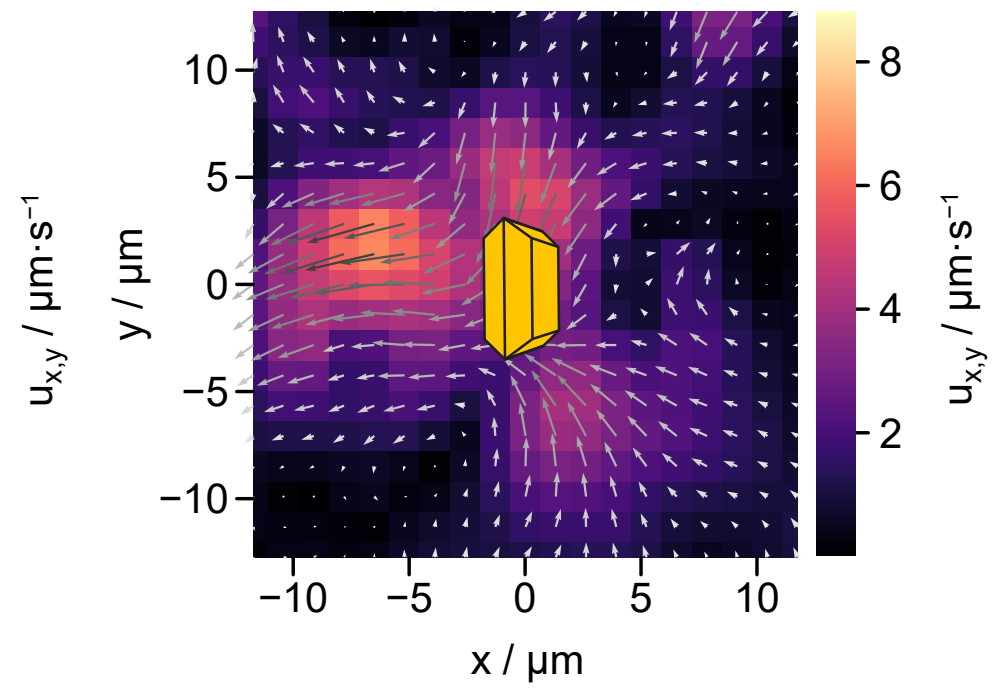

Figure 3: Flow fields of exemplary particles which are oriented in (a) upright and (b) inclined position. Flow direction is shown by arrows and velocity is indicated by the color map with $u_{x, y}=\sqrt{u_{x}^{2}+u_{y}^{2}}$. Inward flows towards oxidative $\{110\}$ facets and outward flows from reductive $\{010\}$ facets can be identified for both particles. 
ric chemical field around single crystalline $\mathrm{BiVO}_{4}$ microswimmers is caused by a combination of chargecarrier separation and self-shadowing. Note, that these analyses illustrate the flows around a three-dimensional object in a two-dimensional field, and neglect influences stemming from additional oxidative particle faces that are oriented towards the glass substrate and the top of the attached particles. Presumably, a similar trend of tracer flow from these oxidative to reductive particle faces is caused here, but further analysis would be necessary for confirmation. However, as the particles only perform motion within the $x$ $y$-plane, analysis of two-dimensional fields gives an overview of the important flows necessary for motion. Also, it should be noted that the flow fields discussed here are obtained from pinned microparticles. When the colloids are in motion, substrate-related flows are expected to impact the flow field.[16] For experimental studies on spherical janus particle microswimmers (PS-Pt), flow fields with the same trend in flow direction, but smaller amplitudes have been obtained for moving microparticles compared to pinned ones.[15] If a similar effect can be expected for the here investigated microswimmers, the flow fields do not only explain the presence of active motion itself, but also the frequently observed tendency for circular trajectories. Enhanced fluid velocities with different amplitudes are caused along both, $x$ and $y$-axis of the particle, which inevitably leads to a torque, especially for upright oriented particles. Inclined microswimmers, however, are often seen to move along rather straight trajectories, which may be caused by the absence of enhanced flow velocities along the self-shadowed side of the particles.

To extract common trends in flow direction, the flow fields of four individual $\mathrm{BiVO}_{4}$ colloids have been

(a) $u_{x}$ along $x$-axis, upright

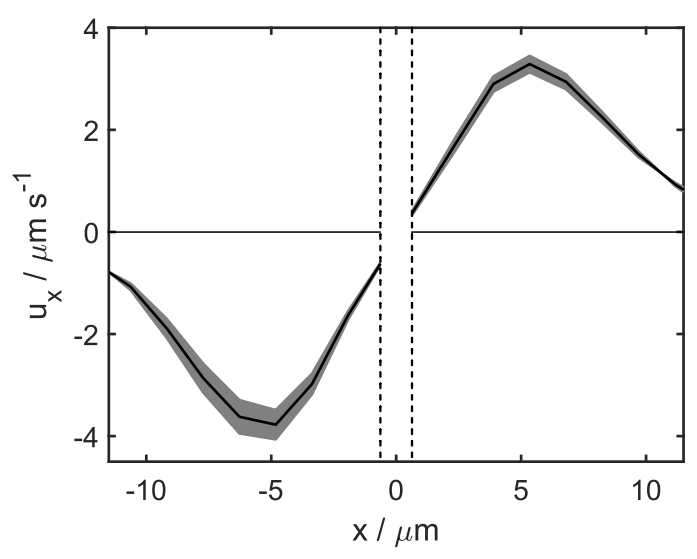

(c) $u_{x}$ along $x$-axis, inclined

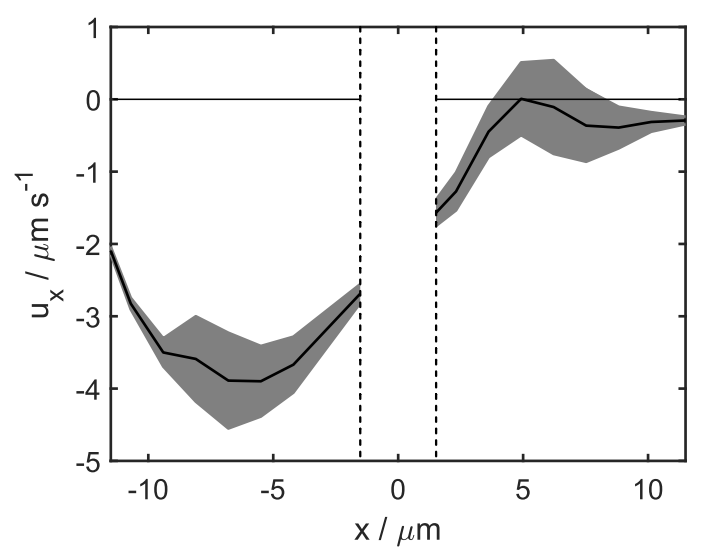

(b) $u_{y}$ along $y$-axis, upright

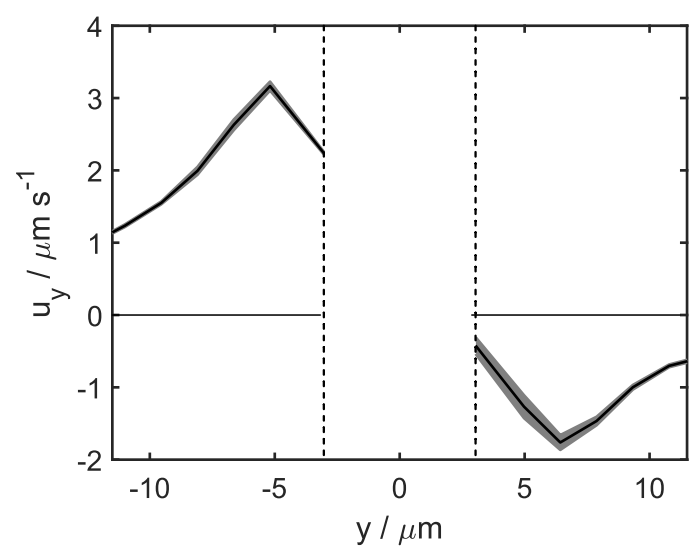

(d) $u_{y}$ along $y$-axis, inclined

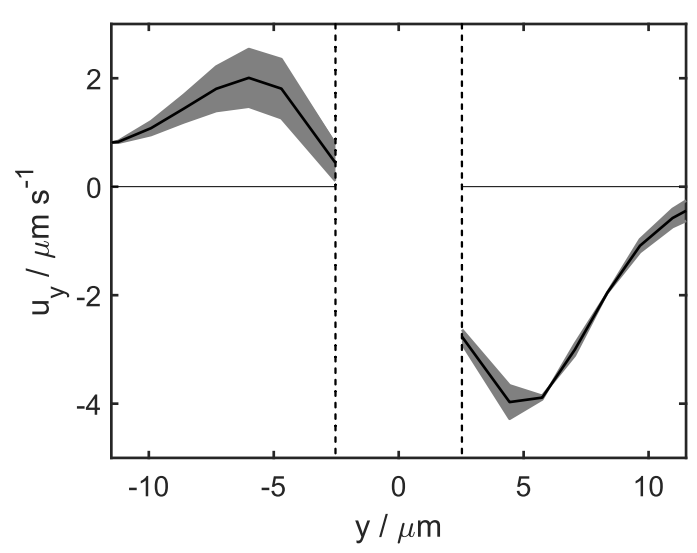

Figure 4: Velocities $u_{x}$ along $x$-axis and $u_{y}$ along $y$-axis of (a), (b) upright and (c), (d) inclined oriented pinned $\mathrm{BiVO}_{4}$ microswimmer. Dashed horizontal lines mark the borders of the pinned colloids. Black solid lines represent the average over two raster rows that intersect the particle center, upper and lower limits of grey colored area are the velocity values of these two rows. One raster row has a width of $1.1 \mu \mathrm{m}$. 
averaged. To ensure comparability, only particles with similar size $(5 \mu \mathrm{m})$ and upright orientation towards the substrate $\left(\alpha=90^{\circ}\right)$ have been considered. The obtained mean flow field is illustrated in Figure 5. Here, it is clearly seen that the trend for the flow direction from oxidative to reductive sides is confirmed. Standard deviations of flow speed magnitude and direction of the mean flow field given in Figure S8 underline that, although different microswimmers cause varying magnitudes of flow speed due to individual synthetic surface defects, comparable flow directions exist. This illustrates, that charge carrier separation due to a surface heterojunction is indeed a sufficient source for an asymmetric chemical gradient around single crystalline $\mathrm{BiVO}_{4}$ microswimmers.

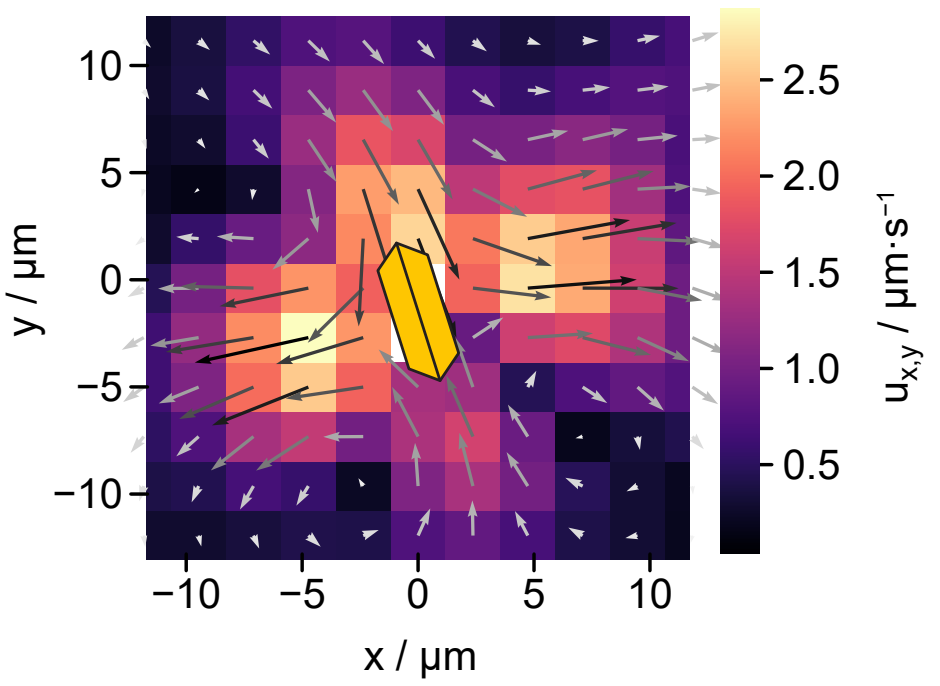

Figure 5: Mean flow field obtained by averaging over the flow patterns from four individual particles with an average size of $5 \mu \mathrm{m}$ and an inclination angle of $90^{\circ}$. A common trend for flows from oxidative to reductive colloid sides is observed.

\section{Conclusion}

Within this manuscript we developed single-component $\mathrm{BiVO}_{4}$ microswimmers with increased activity that rely on an inherent mechanism for charge carrier separation. The clear separation of charge carriers onto different facets enabled us to predict a dominant self-electrophoretic motion mechanism originating from a quadrupolar source field for the circular motion patterns observed under UV illumination in dilute $\mathrm{H}_{2} \mathrm{O}_{2}$ solutions. Particle tracking velocimetry of passive gold tracer particles allowed the illustration of flow fields around individual pinned $\mathrm{BiVO}_{4}$ colloids with different size and inclination angles and the confirmation of our conjectures on the motion mechanism. A common trend for inward flows towards oxidative facets and outward flows from reductive facets can be identified, which resembles the flow profile expected for a pusher. We observe the flows to be asymmetrized by individual surface defects and self-shadowing, resulting in an overlay of the source quadrupole with hydrodynamic effects. Herein we demonstrated that the determination of flow fields can be a powerful tool to investigate and understand the source of colloid motion with various shapes. We visualized that the origin of self-induced fields and the resulting flows are connected by complex interrelations. The spatiotemporal resolution of flows around moving microswimmers, possibly even in three dimensions[30, 31, 32, 33], will enable the profound understanding of motion and interaction behavior in future studies, which is the key to design microswimmers for desired applications such as the investigation of collective behavior and cargo transport.

\section{Experimental Section}

Synthesis of $\mathrm{BiVO}_{4}$ Microparticles: 
For the synthesis of single crystalline $\mathrm{BiVO}_{4}$ microparticles, a procedure by Xie et al. was adapted.[25] In a $40 \mathrm{ml}$ glass vial, $2.425 \mathrm{~g} \mathrm{Bi}\left(\mathrm{NO}_{3}\right)_{3} \cdot 5 \mathrm{H}_{2} \mathrm{O}$ and $0.585 \mathrm{~g} \mathrm{NH} \mathrm{NO}_{3}$ were dissolved in $20 \mathrm{ml} 2 \mathrm{M} \mathrm{HNO}_{3}$ under stirring and ultrasonication. After dissolution, the $\mathrm{pH}$ was adjusted to 2 with $25 \mathrm{wt} \% \mathrm{NH}_{3}$. For shape control, $\mathrm{NaCl}$ with final concentrations of $0.05 \mathrm{M}$ and $0.2 \mathrm{M}$ and sodium dodecyl sulfate with concentrations between $0.05 \mathrm{M}$ and $0.005 \mathrm{M}$ were then added. After stirring for $15 \mathrm{~min}$, the dispersion was transferred to a teflon-lined stainless steel autoclave and left to ripen for $2 \mathrm{~h}$. Subsequently, the synthesis was heated to $200{ }^{\circ} \mathrm{C}$ for $24 \mathrm{~h}$. After cooldown to room temperature, the samples were washed with deionized water and ethanol extensively and dried under air at $70^{\circ} \mathrm{C}$ overnight.

Synthesis of Au Nanoparticles:

$\mathrm{Au}$ nanoparticles were synthesized by a seeded growth method. For the seed synthesis, $500 \mu \mathrm{l} 1 \mathrm{wt} \% \mathrm{HAuCl}_{4}$ were diluted in $50 \mathrm{ml}$ deionized water in a two-necked $100 \mathrm{ml}$ round bottom flask and heated to reflux. Subsequently, $2 \mathrm{ml} 1 \mathrm{wt} \%$ sodium citrate were injected into the solution which was then refluxed for 5 min. After cooldown to room temperature, the seeds were stored under ambient conditions.

For seeded growth, a modified procedure from Liu et al. was followed.[34] In a $10 \mathrm{ml}$ glass vial, $4.8 \mathrm{ml}$ deionized water and $25 \mathrm{\mu l} 1 \mathrm{wt} \%$ sodium citrate were combined under stirring at $1000 \mathrm{rpm}$. Subsequently, $200 \mu \mathrm{l} 1 \mathrm{wt} \% \mathrm{HAuCl}_{4}$ were added, followed by $5 \mathrm{ml} 30 \mathrm{wt} \% \mathrm{H}_{2} \mathrm{O}_{2}$. Then, $5 \mu \mathrm{l} \mathrm{Au}$ nanoparticle seed solution were immediately injected and the solution was stirred for $5 \mathrm{~min}$. For purification, the solution was centrifuged and the supernatant discarded. The obtained Au nanoparticles were dispersed in $1 \mathrm{ml}$ deionized water and stored under ambient conditions.

Powder X-ray Crystallography (XRD):

XRD patterns were acquired using a Bruker $2 \mathrm{D}$ phaser in a $2 \theta$ range of $10^{\circ}-100^{\circ}$, where symmetrical scans were performed. The microparticles were dispersed in ethanol and drop-casted onto a silicon wafer.

Scanning Electron Microscopy (SEM):

For SEM imaging, dilute dispersions of $\mathrm{BiVO}_{4}$ microparticles in ethanol were drop-casted onto silicon wafer pieces and attached to a specimen support with conductive carbon tape. Samples were dried overnight and imaged on a Zeiss Ultra Plus microscope with an acceleration voltage of $3 \mathrm{kV}$.

Transmission Electron Microscopy (TEM):

For TEM imaging, dilute dispersions of Au nanoparticles in ethanol were drop-casted onto carbon-coated copper TEM grids and dried under vacuum overnight. Images were taken on a FEI Tecnai F30 microscope $(300 \mathrm{kV})$.

$U V$-Vis Spectroscopy:

Absorption spectra were obtained on a Cary 600 spectrometer by placing a dilute dispersion of $\mathrm{BiVO}_{4}$ microparticles in deionized water in a quartz cuvette in an integrating sphere. Spectra were measured between $350 \mathrm{~nm}$ and $600 \mathrm{~nm}$.

Light Microscopy Experiments:

For speed analysis, $\mathrm{BiVO}_{4}$ microparticles were dispersed in deionized water and combined with dilute $\mathrm{H}_{2} \mathrm{O}_{2}$ on cleaned $24 \times 24 \mathrm{~mm}$ glass substrates. Therefore, $9 \mu \mathrm{lml}$ particle dispersion was combined with $1 \mathrm{l} \mathrm{ml}$ of $1 \mathrm{wt} \% \mathrm{H}_{2} \mathrm{O}_{2}$ solution, yielding a final concentration of $0.1 \mathrm{wt} \% \mathrm{H}_{2} \mathrm{O}_{2}$. Analysis was performed in an inverted Zeiss Microscope with a flexible Colibri 7 lightsource. Illumination with UV light (385 nm) was focussed by a $63 \times$ air objective to an intensity of $5.6 \mathrm{~W} \mathrm{~cm}^{-2}$. Videos were recorded with $40 \mathrm{fps}$. For flow field experiments, a total volume of $10 \mu \mathrm{l}$ of $0.2 \mathrm{wt} \% \mathrm{H}_{2} \mathrm{O}_{2}$ with $\mathrm{BiVO}_{4}$ microswimmers and $\mathrm{Au}$ tracer particles was applied to a glass substrate and covered by a $18 \times 18 \mathrm{~mm}$ cover slide. Subsequently, the samples were sealed with nail polish and inserted in the inverted Zeiss microscope. Videos were recorded under illumination with UV light that was focussed by a $63 \times$ air objective to $3.8 \mathrm{~W} \mathrm{~cm}^{-2}$ with a frame rate of $40 \mathrm{fps}$.

Video Processing:

For speed and mean-squared displacement analysis of the microparticles, videos were converted to binary images and particles were tracked as Gaussian blobs in each video frame by the Trackmate plugin of the software ImageJ.[35] For data processing, a MATLAB script based on the open-source msdanalyzer 
class was used.[36] Mean speeds were calculated as the unweighted mean of at least 20 individual particle speeds over a time range of $15 \mathrm{~s}$ from different video recordings. The speed is defined as the overall distance travelled by a particle in a given time.

Flow Field Analysis:

For obtaining the flow field near the immobilized microswimmers, the movement of intentionally added Au nanoparticles was recorded with the already mentioned widefield microscope. Localisation of the tracer particles in individual frames as well as the linking of these positions to trajectories over successive images was conducted with the algorithm by Crocker and Gier [37] using the TrackPy package. In order to remove spurious trajectories, only trajectories with a minimum length of 10 frames were considered. Furthermore, erroneous detections inside the microswimmer and particles stuck to it were eliminated by discarding all particles inside a predefined polygon that covered approximately the area of the microswimmer. Subsequently, for every interrogation area in the grid the median of all displacement vectors was calculated and assigned to that position. For grid points without measurement data, the flow field was interpolated using a modified algorithm from the package PTVPy.

The mean flow field was obtained from four different microswimmers. For merging the corresponding flow fields, the coordinate systems of these different measurements were transformed so that all microswimmers exhibited the same position and orientation. Next, the flow field was obtained via interpolation from all trajectories similar to the already described procedure. In order to ensure that all microswimmers contribute an equal amount to the result, the corresponding measurements were weighted inversely proportional to the number of recorded displacement vectors. For every interrogation area, the weighted mean was calculated as well as the corresponding standard deviation (see Supporting Information).

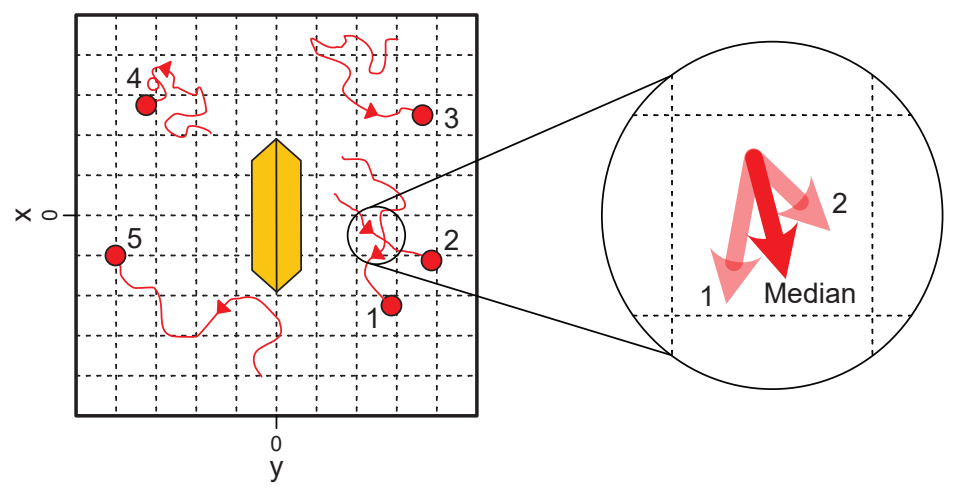

Figure 6: Raster model of PTV processing. In each raster point, the median flow speed and direction is calculated from all tracer particle trajectories present.

\section{Supporting Information}

Supporting Information is available from the Wiley Online Library or from the author.

\section{Acknowledgements}

The authors acknowledge Helena Decker for TEM imaging and William E. Uspal for helpful discussions about the flow fields. This work was supported by a Freigeist fellowship from the Volkswagen foundation (grant number 91619) and by the German Research Foundation (DFG, grant BU 2241/6-1).

\section{References}

[1] M. Safdar, J. Simmchen, J. Jänis, Environmental Science: Nano 2017, 4, 81602.

[2] L. Wang, A. Kaeppler, D. Fischer, J. Simmchen, ACS Applied Materials and Interfaces 2019, 11, 3632937.

[3] B. Jurado-Sánchez, J. Wang, Environmental Science: Nano 2018, 5, 71530. 
[4] J. Parmar, D. Vilela, K. Villa, J. Wang, S. Sánchez, Journal of the American Chemical Society 2018, 140, 309317.

[5] L. Sonntag, J. Simmchen, V. Magdanz, Molecules 2019, 241.

[6] J. Llacer-Wintle, A. Rivas-Dapena, X. Chen, E. Pellicer, B. J. Nelson, J. Puigmartí-Luis, S. Pané, Advanced Materials 2021, 2102049.

[7] J. Wang, W. Gao, ACS Nano 2012, 6, 75745.

[8] E. M. Purcell, American Journal of Physics 1977, 45, 13.

[9] J. L. Moran, J. D. Posner, Annual Review of Fluid Mechanics 2017, 49, 1511.

[10] A. Brown, W. Poon, Soft Matter 2014, 10, 224016.

[11] B. Jurado-Sánchez, M. Pacheco, R. Maria-Hormigos, A. Escarpa, Applied Materials Today 2017, 9 407.

[12] A. M. Pourrahimi, M. Pumera, Nanoscale 2018, 10, 3516398.

[13] A. Zöttl, H. Stark, Journal of Physics Condensed Matter 2016, 28, 25253001.

[14] L. Hecht, J. C. Ureña, B. Liebchen, arXiv 2021, arXiv:2102.13007.

[15] A. I. Campbell, S. J. Ebbens, P. Illien, R. Golestanian, Nature Communications 2019, $10,11$.

[16] A. P. Bregulla, F. Cichos, Journal of Chemical Physics 2019, 151, 4044706.

[17] C. Peng, I. Lazo, S. V. Shiyanovskii, O. D. Lavrentovich, Physical Review E - Statistical, Nonlinear, and Soft Matter Physics 2014, 90, 51.

[18] L. Wang, M. Borrelli, J. Simmchen, ChemPhotoChem 2021, cptc.202100083.

[19] U. Choudhury, D. P. Singh, T. Qiu, P. Fischer, Advanced Materials 2019, 31, 121.

[20] S. Heckel, J. Simmchen, Advanced Intelligent Systems 2019, 11900093.

[21] S. Heckel, J. Grauer, M. Semmler, T. Gemming, H. Löwen, B. Liebchen, J. Simmchen, Langmuir 2020, 36, 4212473.

[22] K. Villa, F. Novotný, J. Zelenka, M. P. Browne, T. Ruml, M. Pumera, ACS Nano 2019, 138135.

[23] R. Li, F. Zhang, D. Wang, J. Yang, M. Li, J. Zhu, X. Zhou, H. Han, C. Li, Nature Communications 2013, 41432 .

[24] G. Liu, J. C. Yu, G. Q. M. Lu, H.-M. Cheng, Chemical Communications 2011, 47, 246763.

[25] S. Xie, Z. Shen, H. Zhang, J. Cheng, Q. Zhang, Y. Wang, Catalysis Science and Technology 2017, 7, 4923.

[26] C. Li, P. Zhang, R. Lv, J. Lu, T. Wang, S. Wang, H. Wang, J. Gong, Small 2013, 9, 233951.

[27] J. L. Moran, P. M. Wheat, J. D. Posner, Physical Review E - Statistical, Nonlinear, and Soft Matter Physics 2010, 81, 61.

[28] Z. Zhao, Z. Li, Z. Zou, RSC Advances 2011, 1, 5874.

[29] Z. Zhao, Z. Li, Z. Zou, Physical Chemistry Chemical Physics 2011, 13, 104746.

[30] J. König, K. Tschulik, L. Büttner, M. Uhlemann, J. Czarske, Analytical Chemistry 2013, 85, 6 3087. 
[31] J. König, K. Tschulik, L. Büttner, M. Uhlemann, J. Czarske, Analytical Chemistry 2013, 85, 6 3087.

[32] F. Bürkle, J. Czarske, L. Büttner, Flow Measurement and Instrumentation 2021, in press.

[33] M. Teich, M. Mattern, J. Sturm, L. Büttner, J. W. Czarske, Optics Express 2016, 24, 2427371.

[34] X. Liu, H. Xu, H. Xia, D. Wang, Langmuir 2012, 28, 3813720.

[35] J. Y. Tinevez, N. Perry, J. Schindelin, G. M. Hoopes, G. D. Reynolds, E. Laplantine, S. Y. Bednarek, S. L. Shorte, K. W. Eliceiri, Methods 2017, 11580.

[36] N. Tarantino, J. Y. Tinevez, E. F. Crowell, B. Boisson, R. Henriques, M. Mhlanga, F. Agou, A. Israël, E. Laplantine, Journal of Cell Biology 2014, 204, 2231.

[37] J. C. Crocker, D. G. Grier, Journal of Colloid and Interface Science 1996, 179, 1298. 